\title{
A microfluidic platform culturing two cell lines paralleled under in-vivo like fluidic microenvironment for testing the tumor targeting of nanoparticles
}

Juan Wei ${ }^{1 \#}$, Lichun Cheng ${ }^{2 \#}$, Jingmin $\mathrm{Li}^{1}$, Yuanchang Liu ${ }^{3}$, Shuqing Yin ${ }^{1}$, Bing $\mathrm{Xu}^{2}$, Dan Wang ${ }^{2}$, Huiyi $\operatorname{Lu}^{2 *}$, Chong Liu ${ }^{1,4^{*}}$

${ }^{1}$ Key Laboratory for Micro/Nano Technology and System of Liaoning Province, Dalian University of Technology, Dalian, China

${ }^{2}$ Department of Pharmacy, The Second Affiliated Hospital of Dalian Medical University, Dalian, China

${ }^{3}$ Department of Mechanical Engineering, University College London, London, UK, NW12BX

${ }^{4}$ Key Laboratory for Precision and Non-traditional Machining Technology of Ministry of Education, Dalian University of Technology, Dalian, China

${ }^{\#}$ These authors contributed equally to this work

*Correspondence

Chong Liu, Key Laboratory for Micro/Nano Technology, Dalian University of Technology, No.2 Linggong Road, Ganjingzi District, Dalian City, Liaoning Province, P.R.C., +86-411-84707946, chong1@dlut.edu.cn; Huiyi Lu, 15541117912@163.com 


\begin{abstract}
Nanoparticles are attractive in medicine because their surfaces can be chemically modified for targeting specific disease cells, especially for cancer. Providing an in-vivo like platform is crucial to evaluate the biological behaviours of nanoparticles. This paper presents a microfluidic device that could culture two cell lines in parallel in in-vivo like fluidic microenvironments and be used for testing the tumor targeting of folic acid - cholesterol - chitosan (FACC) nanoparticles. The uniformity and uniformity of flow fields inside the cell culture units are investigated using the finite element method and particle tracking technology. Hela and A549 cells are cultured in the microfluidic chip under continuous media supplementation, mimicking the fluid microenvironment in vivo. Cell introducing processes are presented by the flow behaviours of inks with different colours. The two cell lines are identified by detecting folate receptors on the cellular membranes. The growth curves of the two cell lines are measured. The two cell lines cultured paralleled inside the microfluidic device are treated with FITC-FACC to investigate the targeting of FACC. The tumor targeting of FACC are also detected by in vivo imaging of Hela cells growth in nude mice models. The results indicate that the microfluidic device could provide a dynamic, uniform and stable fluidic microenvironment to test the tumor targeting of FACC nanoparticles.
\end{abstract}

Key words: Microfluidic; Paralleled introducing; Microenvironment; Targeting; Nanoparticles 


\section{Introduction}

Receptor-mediated nanoparticle drugs are attractive in medicine because they present opportunities to enhance therapeutic efficiency by accumulating drug within the tissue of interest and reducing undesired, off-target effects, especially for cancer [1,2]. Folate receptor (FR) is one of important cancer biomarkers and exceptionally upregulated in many malignant epithelial cells [3]. Folic acid and its conjugates make it possible to differentiate cancer cells by detecting FR expression [4]. Conventionally, the evaluation of targeted drug has been processed in static cell culture plates, which neglects the in-vivo environmental conditions of cells [5]. The in-vivo cells live in a complex microenvironment, including extracellular matrix (ECM), soluble factors, flowing conditions etc. [6,7]. In particular, most of the tissue cells live in environment with interstitial flow, where the fluid flows at a constant, stable and slow velocity $[8,9]$. Interstitial flow has been demonstrated to play an important role in the morphogenesis, function, and pathogenesis of tissues [10,11]. It is therefore important to establish in-vivo like flow models for studying the effects of nanoparticles on tumor cells. Albeit the animal models can be used for evaluating the targeting of nanoparticles or for drug screening, they are time-consuming, expensive, and laborious [12,13] making the demand to develop a biomimetic in vitro model for testing the tumor targeting of nanoparticles critical.

Microfluidic technology is a crucial tool to provide in-vivo like microenvironments for in vitro cells and is important to improve the reliability of cell chemotaxis analysis, drug screening and medical diagnostics [14-16]. Taking the advantages of microfluidic chips, such as the considerable reduction of reagent consumption, the continuous supply of fresh media containing oxygen and nutrients at a controlled flow rate and the precise control of fast analyses, a number of biomimetic in vitro models have been developed for drug research [17-19]. Wang et al. have designed a microfluidic chip to establish an oxygen gradient and applied to study cytotoxicity of two anticancer drugs on A549 cells [20]. An et al. have developed a microfluidic cell culture array to perform combinational chemotherapy on prostate cancer PC3 cells [21]. These microfluidic devices could not culture two or more types of cell lines simultaneously. Jastrzebska et al. have proposed a multi-functional microsystem for cells migration analysis and evaluation of photodynamic therapy 
procedure in co-culture, mono-culture as well as mixed culture $[22,23]$. Zhang et.al have developed an in vitro liver model in a microfluidic device to imitate and detect prodrug metabolism. HepG2 cells and MCF-7 cells are co-cultured in the device [24]. Jarvis et al. have designed a microfluidic co-culture device to assess penetration of nanoparticles into cancer cell mass. The co-culture microfluidic device characterized with an inner tissue culture chamber and two flanking outer vascular channels connected to the tissue chamber via micron sized pores [25]. Ai et al. have proposed a microfluidic device consists of two sets of thin interconnecting grooves to culture the macrophage cells and myocardial cells [26]. These devices can culture two cells in 2D simultaneously and be used for testing drug targeting. However, the large volume of medium flowing through the culture chamber may induce high shear stress, which may have a detrimental effect on active cells. The stability and uniformity of flow fields inside the culture chamber may need to be further improved. 3D co-culture, such as spheroids, multilayers and scaffolds have been created in microsystems recently [27-29]. Zuchowska et al. have proposed a microfluidic 3D co-culture model based on spheroids for studying the effects of nanoencapsulated meso-tetraphenylporphyrin [30]. Ozkan et al. have developed a 3D tissue-on-a-chip platform for dynamic determination of nanoparticle transport and toxicity [31]. Nagaraju et al. have proposed a microfluidic tumor-vascular model to study breast cancer cell invasion and intravasation [32]. These microfluidic devices can achieve cell-cell communication in $3 \mathrm{D}$ co-culture. Cell-cell communication is a very important, but complex physiological behavior in life entity $[33,34]$. In this way, there are many complex influence factors of efficiency of drug. Thus, the experimental results may be various. Besides, due to the porous structure of the 3D culture system, the flow field distribution may be non-uniform and quantitative analysis may be complicated.

In this work, a robust microfluidic chip that could culture two cell lines in parallel under the same fluid microenvironment has been designed. The uniformity and stability of flow fields inside the culture chambers have been investigated using the finite element method (FEM) together with the wall shear stress. Cell introducing processes are presented by the flow behaviours of inks with different colours, and Hela and A549 cells are cultured in the microfluidic chip. The two cell lines are identified by detecting folate receptors on the cellular membranes with the growth curves of 
the two cell lines been measured. The targeting of FACC on the two cell lines are investigated by treating the cells with FITC-FACC. The tumor targeting of FACC are also detected by in vivo imaging of Hela cells growth in nude mice models.

\section{Material and methods}

\subsection{Design of the microfluidic device}

To culture two cell lines paralleled, an axisymmetric structure of the microfluidic chip is designed (as shown in Fig.

1a). The symmetry axis goes through the center of medium inlet and center of guide hole, which divides the microchannels into left and right branches. The microfluidic chip consists of four layers with the top layer being the valve control layer, including twelve through-holes matched with twelve plugs. The fluid flow into the chip could be controlled by adjusting the state of plugs using mechanical force. The second layer of the microfluidic chip is the cell introducing layer consisting of two cell introducing channels, seven through-holes matched with the medium inlet, two cell inlet, two cell outlets, two medium outlets, a guide hole matched with plug 1 . The guide hole locates at the intersection of the left and right branches. When introducing cells, plug 1 is squeezed into the guide hole. Then the connection between the left branch and right branch is interdicted, which can avoid the mixing of two cell lines. When infusing cell culture medium, plug 1 is pulled out, plug 5-8 are squeezed into the corresponding through-holes, medium could flow into the left branch and right branch equally. The third layer is the intermediate layer, which is a 0.1 mm-thick cover plate with through-holes aligned at the reservoir and the cell culture chambers. The film is elastic, which could be deformed by the squeezing of plugs. The cell suspension can be accurately introduced into cell culture chambers. The bottom layer is the cell culture layer, including the reservoir, main channel, bifurcated channels and cell culture units. The structures of six cell culture units are uniform.

In previous works, the existence of multiple flow paths around the cell culture chamber has been demonstrated to be useful for constructing a uniform and stable flow fields inside the chamber [35,36]. In this work, microgaps around the cell culture chamber has also been designed (as shown in Fig. 1b). The shape of the culture chamber is octagon 
circumscribed with an ellipse. The major axis of the ellipse is $2 \mathrm{~mm}$ and the minor axis is $1 \mathrm{~mm}$. The depth of culture chamber, microgaps and channels are all $100 \mu \mathrm{m}$. The circumferential width of microgap is $30 \mu \mathrm{m}$. The culture medium infused from the medium inlet flows into the left and right main channels, then goes through bifurcated channel I, bifurcated channel II, flows into and out of the cell culture chamber through the microgaps by convection and diffusion. The main channel has a width of $300 \mu \mathrm{m}$, the bifurcated channel I has a width of $147 \mu \mathrm{m}$ and the bifurcated channel II has a width of $70 \mu \mathrm{m}$. Both of the main channel and bifurcated channel I are divided into two uniform branches. The hydraulic diameters of the main channel and the bifurcated channels obey Murray's law, which mimics the structural arrangement of blood vessels transporting nutrients to cells. The existence of hierarchical bifurcation microchannels and micropillar array can greatly reduce the flow velocity of the culture medium and make the culture medium flow into and out the culture chamber in many directions, which is in favor of maintaining the uniformity and stability of flow fields inside culture chamber.

The axisymmetric structures of the microfluidic chip can achieve the paralleled introducing of two cell lines based on flow balance [23]. Since the liquid volume of the microfluidic chip is extremely small (about $5 \mu \mathrm{l}$ ), a slight disturbance (e.g. the progressive error of pump) would break the flow balance at the position of connection between the left branch and right branch. Thus, a controllable valve is needed and a holder is designed to fix the microfluidic chip and control the state of plugs as shown in Fig. 1c. The holder consists of an upper plate, four adjustable bolts and a bottom plate. In order to fix the positions of plugs and tubes, seven holes aligned at the inlets and outlets of microfluidic chip are designed. The states of plugs can be controlled by adjusting the bolts, which subsequently controls the fluid flow inside the microfluidic chip.

\subsection{Fabrication of the microfluidic device}

Four layers of the microfluidic chip are fabricated with polydimethylsiloxane (PDMS, Sylgard 184, Dow Corning) by replicating structures on the masters [36]. The volumes of PDMS for the valve control layer, the cell culturing layer, the transition layer and the cell culture layer are $16.2 \mathrm{ml}, 0.8 \mathrm{ml}, 0.8 \mathrm{ml}$ and $8.1 \mathrm{ml}$, respectively. The masters of the valve 
control layer and the cell culture layer with PDMS are put in self leveling drying baker for $0.5 \mathrm{~h}$ to level the PDMS. The masters of the cell introducing layer and the transition layer with PDMS are put in the spin coater to accelerate the flow of PDMS, then put in the self-leveling drying baker. The polymers are oven-cured $\left(80^{\circ} \mathrm{C}, 1 \mathrm{~h}\right)$. After cooling, the PDMS layers of the valve control layer and the cell culture layer are gently peeled from the masters and trimmed to size. The PDMS piece of the cell introducing layer and the transition layer are thin, and to avoid the PDMS pieces being teared the PDMS pieces are peeled from the master after being bonded. The PDMS pieces are treated by oxygen plasma for 50 s, aligned using the visual alignment equipment and bonded irreversibly [37].

The plugs used for controlling the flow are fabricated by infusing PDMS into pipette tips, oven-curing PDMS and cutting the pipette tips into suitable length. The upper plate and bottom plate are incised by a $\mathrm{CO}_{2}$ laser ablation system (JinBoshi, JBSCO2-50) into designed sizes [38].

\subsection{Simulation of cell culturing process}

To verify that two cell lines could be cultured paralleled in the chambers, the cell introducing and culturing processes have been presented visualized by the flow behaviours of inks (SHANGHAI HERO co., ltd, shanghai) with different colours. The switching of channels is controlled by plugs. The blue ink represents Hela cell suspension, the red ink represents A549 cell suspension and the yellow ink represents culture medium. When introducing the cell suspensions, plug 1 inside the through-hole is pressed while the medium inlet and medium outlets are being blocked. When the culture medium is introduced, plug 1 inside the through-hole is taken out, the cell introducing channels are interdicted by pressing plug 5-8, at the same time the cell inlets and outlets are blocked.

\subsection{Evaluation of flow fields inside the microfluidic chip}

In order to evaluate the flow fields inside the culture chambers, the Multiphysics Software COMSOL (version 5.3, Burlington, MA) is used. The six chambers are labelled as R1, R2, R3, L1, L2, L3, respectively. The 3D finite element model based on laminar Poiseuille flow is developed, and the flow field is obtained by solving the Navier-Stokes equation. Water under $37^{\circ} \mathrm{C}$ is selected as the fluid material [36,39]. A sinusoidal variation $(v=925+462.5 * \sin (\pi * t)$, 
$\mu \mathrm{m} / \mathrm{s}$ ) is set as the medium inlet velocity. Other simulation conditions are configured as follows: 1) zero traction is set at the outlet and no slip boundary is set on the surface, 2) the time step is $(0,0.1,2)$ and 3$)$ the bottom of the culture chamber is set as $\mathrm{z}=0 \mathrm{~mm}$ plane. To investigated the uniformity of flow fields inside the six chambers, the area average velocities of each culture chamber are calculated when $\mathrm{t}=2 \mathrm{~s}, \mathrm{z}=0.05 \mathrm{~mm}$. To study the uniformity of the flow fields inside the single chamber, the velocities along four diagonal lines of the R1 chamber are calculated when $\mathrm{t}=2 \mathrm{~s}, \mathrm{z}=0.05$ $\mathrm{mm}$. To study the stability of flow fields inside the cell culture chamber, the average velocity inside the R1 chamber under varying medium inlet velocity is calculated at $\mathrm{z}=0.05 \mathrm{~mm}$. Shear stresses induced by fluid flow affect the proliferation, differentiation and expression of cells [40,41]. It is important to limit shear stress to a moderate level $\left(10^{-2}-10^{-4}\right.$ dyne $\left./ \mathrm{cm}^{2}\right)[42]$. Since the cells attach to the bottom of culture chambers, the wall shear stresses are investigated when $\mathrm{t}=2 \mathrm{~s}, \mathrm{z}=0 \mathrm{~mm}$.

The particle tracking technology is used to further evaluate the flow fields in the chambers, and Polystyrene (PS) microbeads (Sphere Scientific Co., Ltd., China) are used as tracking particles. The experiment processes are carried out according to the protocols described in our previous work [35]. The PS microbead suspension with concentration of $0.01 \%$ is perfused into the microfluidic devices by a Harvard PHD-Ultra syringe pump (Harvard Apparatus, Inc., USA) , and the movements of 25 microbeads within the chambers are observed by an inverted microscope (Olympus IX71, Japan), recorded and dealt with Image Pro Plus 6.0 (Media Cybernetics Inc., USA). Constant flow rate of $50 \mu \mathrm{l} / \mathrm{h}, 70$ $\mu \mathrm{l} / \mathrm{h}, 100 \mu \mathrm{l} / \mathrm{h}, 120 \mu \mathrm{l} / \mathrm{h}$ and $150 \mu \mathrm{l} / \mathrm{h}$ are set on the pump, respectively.

\subsection{Cell culture}

The A549 cells and Hela cells (Cell Bank of the Chinese Academy of Sciences, Shanghai, China) are cultured simultaneously in the same microfluidic chip. The device is sterilized and coated with fibronectin according to previous works [35,36]. Before introducing the cells, the microchannels are filled with culture medium containing $89 \%$ DMEM, $10 \%$ FBS and 1\% PS (Gibco, USA) to remove the surplus proteins and gas. All of the inlets and outlets are sealed by medium to isolate the microchannels with atmosphere. A549 cells and Hela cells are detached from the cell culture dish 
with $0.5 \%$ trypsin respectively, suspended in the culture medium, counted with the hemocytometer, and diluted into the same densities of $1 \times 10^{6}$ cells $/ \mathrm{ml} .50 \mu \mathrm{l}$ cell suspensions of Hela and A549 cells are injected into the chip from two cell inlets respectively, and the microfluidic chip is placed in static in a $5 \% \mathrm{CO}_{2}$ incubator at $37{ }^{\circ} \mathrm{C}$ for $6 \mathrm{~h}$. Then the perfusion of culture medium is started with a flow rate of $100 \mu \mathrm{l} / \mathrm{h}$ and completed inside the incubator. The culture medium in the syringe is changed every 24 hours. Observations of cell cultures are carried out using an inverted fluorescence microscope (Leica, Germany, DMD000B) every 24 hours. The pictures are analyzed by using Image Pro Plus 6.0 and the cell numbers are counted. The relative growth rate $\left(\left(\mathrm{N}_{\mathrm{A}}-\mathrm{N}_{\mathrm{B}}\right) / \mathrm{N}_{\mathrm{B}}\right.$ is calculated, where $\mathrm{N}_{\mathrm{A}}$ is the cell number at time $\mathrm{A}, \mathrm{N}_{\mathrm{B}}$ is the cell number at time $\mathrm{B}$, and $\mathrm{A}>\mathrm{B}$ ).

\subsection{Measurement of folate receptors on cell membrances}

$50 \mu \mathrm{l}$ cell suspensions of Hela and A549 cells with densities of $1 \times 10^{6}$ cells $/ \mathrm{ml}$ are injected into the same chip simultaneously from two cell inlets. Folate receptors on membrance of Hela and A549 cells are measured after culturing for $78 \mathrm{~h}$ inside the microfluidic chip. $100 \mu \mathrm{l}$ rabbit monoclonal anti-human folate receptor (1:500) antibody (Abcam, USA) is infused into the chambers and incubated for $2 \mathrm{~h}$ at $37^{\circ} \mathrm{C}$. The medium inlet and outlets are sealed by culture media. Then $100 \mu \mathrm{l}$ TRITC-labeled goat anti-rabbit immunoglobulin antibody (1:150, Bioworld, USA) is infused into the chip and incubated for 20 min. The staining solution is removed and washed three times with PBS. The fluorescence in each sample is monitored by an inverted fluorescence microscope (Leica, Germany, DMD000B).

\subsection{Evaluation of tumor targeting of FACC nanoparticles}

The folic acid-cholesterol-chitosan (FACC) nanoparticles have been prepared through aminoacylation reaction and synthesized by lyophilization according to the protocols described in our previous work [43]. The FACC nanoparticles present to be spherical morphology with particle size about $100 \mathrm{~nm}$. In order to test the distribution of FACC nanoparticles in Hela and A549 cells, FITC-conjugated FACC nanoparticles are created according to the published protocol [44]. Hela (folate receptor-positive) and A549 (folate receptor-negative) cells are seeded into the microfluidic chip at a density of $1 \times 10^{6}$ cells $/ \mathrm{ml}$ and incubated for $78 \mathrm{~h}$ at $37^{\circ} \mathrm{C}$. After culturing for $78 \mathrm{~h}$, FITC-FACC nanoparticles 
are infused into the microfluidic chip. The two cell lines are incubated by FITC-FACC nanoparticles for $10 \mathrm{~min}$ and be washed by PBS. The fluorescence in each sample is monitored by an inverted fluorescence microscope (Leica, Germany, DMD000B).

In order to further demonstrate the targeting of FACC nanoparticles, the distribution of FACC in nude mouse and different organs are investigated. The athymic nude mice used in this study are 4-6 weeks old and weigh at about 18-22 g. Each mouse is anesthetized with isoflurane before being injected subcutaneously under left armpit with $0.1 \mathrm{~mL}$ sterile PBS containing $3 \times 10^{6}$ Hela cells. After the mean diameters of tumor mass reach $2-5 \mathrm{~mm}$ in volume, the mice are used for in vivo imaging studies. All animal experiments are carried out in compliance with the Animal Management Rules of the Ministry of Health of the People's Republic of China (document no. 55, 2001). All experiments are approved by Dalian Medical University Licensing Committee. The processes of the experiment are shown as follow. First, 12 athymic nude mice bearing tumor are evenly and randomly divided into two groups. Second, each Hela tumor-bearing mouse in group 1 is injected with $10 \mu \mathrm{I}$ DIR derivative-loaded FACC nanoparticles and each Hela tumor-bearing mouse in group 2 is injected with free DIR derivative. Third, the dynamic behavior of DIR-FACC nanoparticles are monitored by the Living Image 3.1.0 software (Caliper Life Sciences, Hopkinton, MA, USA). The subjected mouse is firstly anesthetized and imaged at predetermined intervals $(3 \mathrm{~h}, 6 \mathrm{~h}, 12 \mathrm{~h}, 24 \mathrm{~h}, 48 \mathrm{~h}$ and $72 \mathrm{~h})$ post-injection. All fluorescence images are acquired with $\mathrm{c} 1-\mathrm{s}$ exposure $(\mathrm{f} / \mathrm{stop}=4)$. Besides, to confirm the in vivo distribution of FACC nanoparticles, mice are sacrificed $72 \mathrm{~h}$ post injection. The excised organs and tissues (heart, liver, spleen, lung, kidney and tumor) are also non-invasively imaged by the self-built NIR imaging system.

\section{Results and discussion}

\subsection{Cell culturing process simulated by the flow of inks}

In order to demonstrate the process of cell culturing, the flow behaviours of inks (SHANGHAI HERO co., ltd, shanghai) with different colours are presented. Different colours represent different processes with the blue ink representing Hela cell suspension, the red ink representing A549 cell suspension and the yellow ink representing culture 
medium. As stated above, the holder is used to fix the microfluidic chip and subsequently controls the state of plugs (as shown in Fig. 2a). The precondition of culturing Hela and A549 cells paralleled is introducing the two cell lines without mixing. When introducing the two cell lines, the medium inlet and medium outlets are blocked off by pressing down Plug 1. Hela cells are introduced into the left branch of culture chambers from cell A inlet, and A549 cells are introduced into the right branch of culture chambers from cell B inlet. The redundant cell suspensions spill from cell outlets. Fig. $2 \mathrm{~b}$ shows that the left and right branches are filled with blue and red inks separately, which indicates two types of cell suspensions are mixed. After the cells attaching to the bottom of culture chamber, the process of infusing the culture medium into the chip is also simulated (as shown in Fig. 2c). The cell inlets and cell outlets are blocked off by pulling out Plug 1. Culture medium is then infused into the medium inlet. The yellow ink flows almost simultaneously through the left and right branches. The result indicates that the two types of cells could be cultured in the same fluid environment. Fig. $2 d$ shows the microfluidic chip is full with yellow ink, which indicates that the culture chambers are filled with culture medium and the waste medium is able to flow out from medium outlets. The results show that the microfluidic chip can be used for culturing two types of cells paralleled and testing the tumor targeting of nanoparticles.

\subsection{Numerical simulation of the flow field}

The flow fields inside the culture chambers are studied using COMSOL Multiphysics software. The uniformity of the velocities of the six chambers are investigated when the velocity of medium inlet is $925 \mu \mathrm{m} / \mathrm{s}$ and the cross profile is $\mathrm{z}=0.05 \mathrm{~mm}$ (as shown in Fig. 3a). It can be seen from the colour bar that the velocity inside each cell culture chamber are almost the same intuitively. The area average velocities of the six chambers have also been calculated to investigate the uniformity of the six chambers (as shown in Fig. 3b). The area average velocities of the six chambers are all 65.9 $\mu \mathrm{m} / \mathrm{s}$. The results indicate that the six culture chambers have exactly the same flow microenvironment and can culture two cell lines in parallel, which can be used for testing the targeting of nanoparticles.

A uniform supply of culture medium is required to guarantee the same fluid environment for all the cells in one 
culture chamber [45]. To study the uniformity of the velocities inside one chamber, the flow fields inside R1 chamber are investigated when velocity of medium inlet is $925 \mu \mathrm{m} / \mathrm{s}$ and cross profile is $\mathrm{z}=0.05 \mathrm{~mm}$ (as shown in Fig. $3 \mathrm{c}$ ). The black lines are the streamlines. Inside the chamber, the colour bar illustrates that the velocities of most of area are the same; whereas the velocities near the microgaps are bigger. The velocity profiles along the lines A-A', B-B', C-C' and D-D' indicate that nearly $86.7 \%$ of velocities are uniform. Most of cells attach to the area of internal ellipse of the chamber (yellow line in Fig. 3c). The average and standard deviation of the velocities within the yellow line is calculated to be $48.7 \pm 2.1 \mu \mathrm{m} / \mathrm{s}$, which further indicates that the velocities inside the culture chamber are uniform.

A stable fluid environment can guarantee the balance of nutrition and metabolic waste in a manner similar to the situation in vivo [46]. A sinusoidal variation of velocity $(\mathrm{v}=925+462.5 * \sin (\pi * t) \mu \mathrm{m} / \mathrm{s})$ is set in the medium inlet as an external disturbance to investigated the stability of flow fields inside the culture chamber. Since the flow fields of the six chambers are the same, it is reasonable to only investigate one of the six chambers'. The velocity variations with R1 chamber are investigated. Fig. $3 \mathrm{~d}$ shows that the average velocity inside the chamber has a fluctuation within $5.7 \%$ of the change in inlet velocity. The results indicate that the designed structures can share the fluctuation of the fluid and as a result maintain a stable fluid environment inside the culture chamber.

Since the cells are attached to the bottom of chambers, the wall shear stress inside the R1 chamber has also been investigated (as shown in Fig. 3e). It can be seen that the average wall shear stress is $1.87 \times 10^{-2} \mathrm{dyne} / \mathrm{cm}^{2}$, which belongs to the level of interstitial flow [42] and is similar with in-vivo condition.

\subsection{Flow behavior of tracking particles inside the culture chambers}

Flow of the PS microbeads inside the chambers are presented and recorded as shown in Fig. 4a. Trajectories of 25 PS microbeads inside the six chambers are shown in supplementary information Fig. S1. In terms of the experimental results of tracking particles, the blue lines in Fig. 4b show the trajectories of the PS microbeads inside the R3 chamber, which are nearly in accordance with the fluid streamlines in the simulation model.

To estimate the uniformity of velocities inside the culture chambers, the average velocities of the 25 microbeads 
distributed uniformly inside the R1, R2, R3, L1, L2 and L3 chambers are measured, which are $42.17 \pm 3.21 \mu \mathrm{m} / \mathrm{s}$, $41.37 \pm 2.62 \mu \mathrm{m} / \mathrm{s}, 44.63 \pm 4.11 \mu \mathrm{m} / \mathrm{s}, 43.92 \pm 4.03 \mu \mathrm{m} / \mathrm{s}, 42.10 \pm 3.07 \mu \mathrm{m} / \mathrm{s}$ and $43.33 \pm 2.93 \mu \mathrm{m} / \mathrm{s}$ respectively (Fig. 4c). The average velocities and standard deviations inside the six chambers are shown in Fig. 4c. The results indicate that the average velocities and standard deviations inside the six chambers are similar, which means the same microenvironment can be formed in the six cell culture units of the chip. Besides, the microfluidic chip can form a uniform fluid microenvironment inside one culture chamber. Such a phenomenon is due to the reason that the microgaps provide alternative directions for fluid flow, consequently enhancing the uniformity of fluid flow in the chamber.

To study the stability of velocity inside the culture chambers, five constant flow rates of $50 \mu 1 / \mathrm{h}, 70 \mu \mathrm{l} / \mathrm{h}, 100 \mu \mathrm{l} / \mathrm{h}$, $120 \mu \mathrm{l} / \mathrm{h}$ and $150 \mu \mathrm{l} / \mathrm{h}$ are initiated in the medium inlet. Fig. $4 \mathrm{~d}$ shows the variation of average velocity in the medium inlet and six chambers. The variation of average velocity inside chamber is about $6.5 \%$ of velocity variation in medium inlet. The results show that the flow fields within the six chambers are stable owing to the reason that the redundant microchannels can provide alternative routes to accommodate external disturbances and maintain the stability of flow fields.

\subsection{Hela and A549 cells cultured inside the microfluidic chip}

Densities of Hela and A549 cells. To study the effects of fluid flow on cell proliferation, the cell densities of Hela and A549 cells cultured in microfluidic chips have been measured within $102 \mathrm{~h}$. The two cell lines cultured in culture plates are used as comparisons. Fig. 5a shows the growth curves of Hela and A549 cells cultured in plates and chips respectively. A dramatic increase of the densities of the two cell lines can be observed between $30 \mathrm{~h}$ and $78 \mathrm{~h}$, which are consistent with standard s-shape cell growth curves. During the culturing time, the relative growth rates of Hela cells in plate, A549 cells in plate, Hela cells in chip and A549 cells in chip are $465.2 \%$ and $368.0 \%, 463.1 \%$ and $366.0 \%$. As the culture time under $54 \mathrm{~h}$, the relative growth rates of Hela cells in plate and A549 cells in plate are $194.0 \%$ and $138.5 \%$, which are higher than these cells in chips (Hela cells in chip is $110.8 \%$ and A549 cells in chip is $82.2 \%$ ). When the culture time is above $54 \mathrm{~h}$, the relative growth rates of Hela cells in chip and A549 cells in chip are $136.1 \%$ and $155.7 \%$, 
which is evidently higher than that in the culture plate (Hela cells in plate is $92.3 \%$ and A549 cells in plate is $122.0 \%$ ).

The cells in plates grow faster than those in chips at the initial stage, which may be because that the static fluid environment make for the accumulating of endogenous growth [47]. With the increase of culturing time, the cells in chips grow faster than those in plate and one of the possible causes is that the dynamic fluid environment can provide balance between the accumulating of endogenous growth factor and the removing of metabolic waste. The results indicate that the microfluidic chip is beneficial to the proliferations of Hela and A549 cells.

Distribution of folate receptors on cell membrane. In order to distinguish the Hela and A549 cells cultured inside the chambers, the distribution of folate receptors on cell membranes are investigated (as shown in Fig. 5b and Fig. 5c). The two cell lines cultured in the same microfluidic chip are treated with rabbit monoclonal anti-human folate receptor and TRITC-labeled goat anti-rabbit immunoglobulin antibody after culturing for $78 \mathrm{~h}$. Fig. $5 \mathrm{~d}$ shows the fluorescence intensity of TRITC. It can be seen that the fluorescence intensity in Hela cells is higher than that in A549 cells, indicating that the Hela cells express more folate receptors than A549 cells. The results indicate that the microfluidic chip is suitable for testing the folic acid (FA) loaded targeting nanoparticles. Besides, the using of microfluidic chip could reduce the reagent consumption and improve the experiment throughput.

In vitro cellular uptake of FACC nanoparticles. In order to test the distribution of FACC nanoparticles in Hela and A549 cells, FITC-conjugated FACC nanoparticles are synthesized. After culturing for $78 \mathrm{~h}$, FITC-FACC nanoparticles are infused into microfluidic chip. The two cell lines are incubated by FITC-FACC nanoparticles for 10 min and be washed by PBS. As shown in Fig. 5e, the intracellular areas of Hela cells present green fluorescence. While the green fluorescence inside A549 cells are weak and the membranes of A549 cells present green fluorescence (as shown in Fig. 5f). The intracellular uptake efficiency of FITC-FACC in Hela cell is much higher than that in A549 cell, indicating that the FACC nanoparticles have targeting on Hela cells. The results also prove that the microfluidic chip can be used for testing tumor targeting of nanoparticles.

\subsection{In vivo tumor targeting of FACC nanoparticles}


To further demonstrate the tumor targeting of FACC nanoparticles, the distribution of FACC in nude mouse and different organs are investigated. Fig. 6a shows the distribution of DIR-FACC in nude mouse varying with time. The DIR solution is used as a comparison. The results indicate that the injection with FACC nanoparticles has stronger fluorescence intensity on the tumor parts compared to the injection with DIR solution. Fluorescence intensity on the tumor parts of the two groups decrease with time. The decrease rate of fluorescence intensity in DIR-FACC Nps group is lower than that in DIR solution group, indicating that the DIR-FACC in vivo has slow release characteristic. After $72 \mathrm{~h}$, the mice are sacrificed and the excised organs and tissues (heart, liver, spleen, lung, kidney and tumor) are also imaged by the self-built NIR imaging system (as shown in Fig. 6b). It can be seen that the fluorescence in DIR-FACC Nps group mainly concentrate in tumor, liver, lung. The fluorescence in DIR solution group mainly concentrate in the liver, lung, and less distribution of fluorescence in the tumor tissue. The results further indicate that FACC nanoparticles have targeting on folate receptor positive tumor.

\section{Conclusions}

A microfluidic chip has been developed to culture two cell lines paralleled in the in-vivo liked fluidic microenvironment. The chip features with six cell culture units distributed symmetrically on the left and right branches. The simulation results show that the designed chip can provide a uniform and stable flow fields inside the culture chambers. In addition, the wall shear stress on the bottom of culture chambers could be controlled in an interstitial flow level. The imitation of cell introducing processes demonstrate that the chip could achieve sampling of two cell lines simultaneously without mixing. The densities of Hela and A549 cells further prove that the chip is favorable to cell culture. The fluorescence intensities inside Hela and A549 cells treated with FITC-FACC indicate the targeting of

FACC. The in vivo imaging of Hela cells growth in nude mice models has also demonstrated the tumor targeting of FACC. In conclusion, the designed microfluidic chip could provide an in vivo like fluidic microenvironment and be used as an in vitro model for testing the tumor targeting and toxicities of nanoparticles.

\section{Acknowledgements}


This work is supported by National Natural Science Foundation of China (51875084), Key Laboratory Open Project Foundation of Dalian University of Technology (JMTZ202002), Science and Technology Planning Project of Dalian (1712032). 

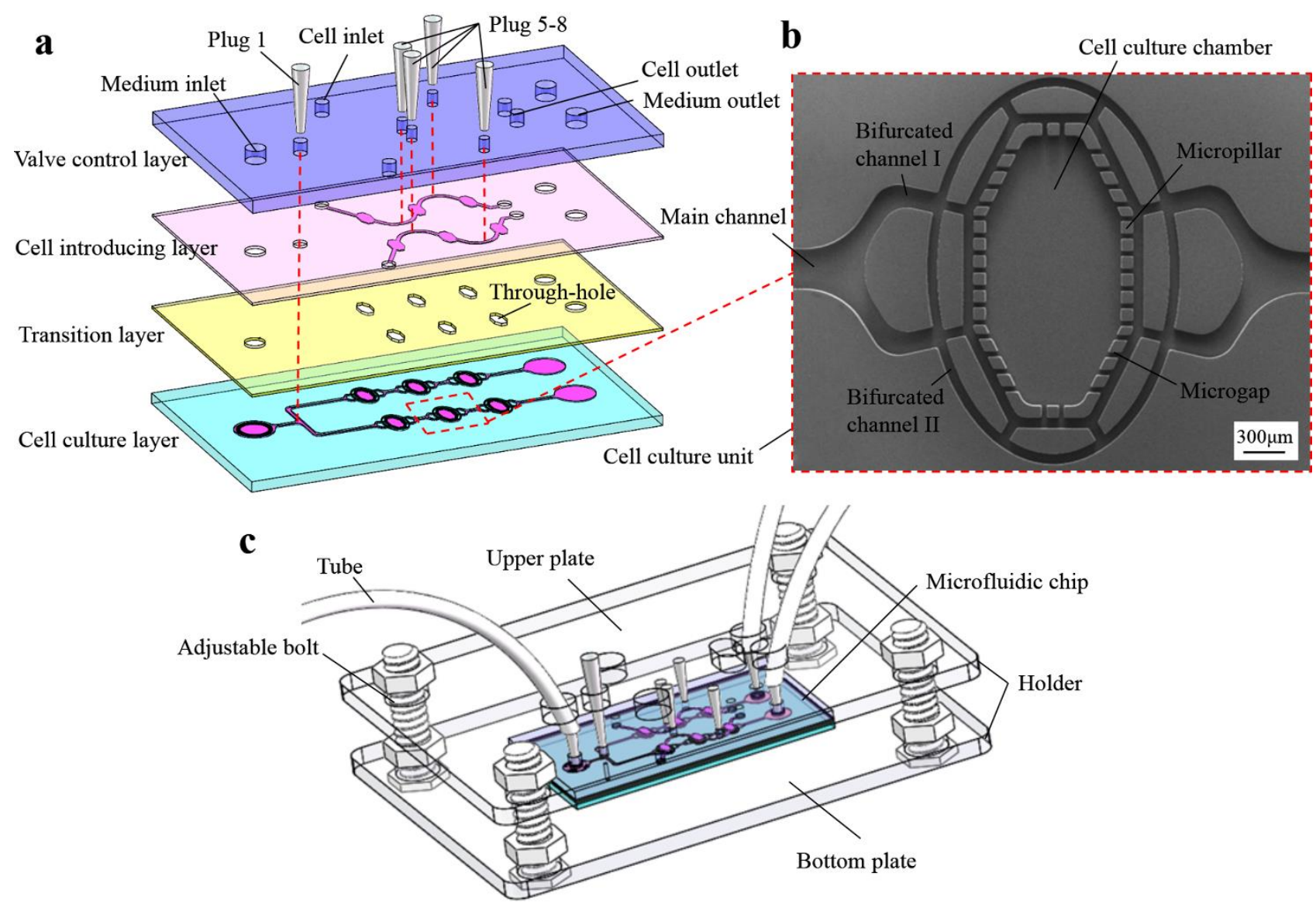

Fig. 1 The structure of the microfluidic chip. (a) Schematic illustrations of the 3D layered structure of the microfluidic chip, including valve control layer, cell introducing layer, transition layer and cell culture layer. (b) SEM image of the cell culture unit. 

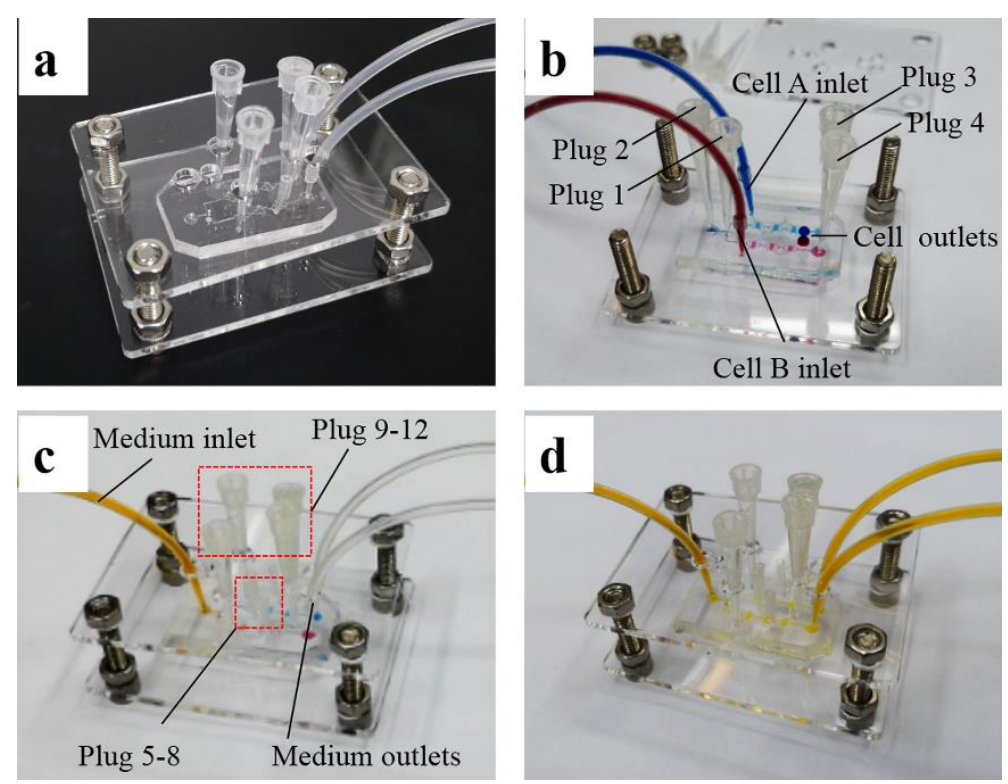

Fig. 2 Simulation of cell culturing process. (a) The object of the whole device. (b) The simulation of cell introducing process. (c) The simulation of culture medium infusing process. (d) The simulation of process that the microfluidic chip is full with culture medium. The blue ink represents Hela cell suspension, the red ink represents A549 cell suspension and the yellow ink represents culture medium. 

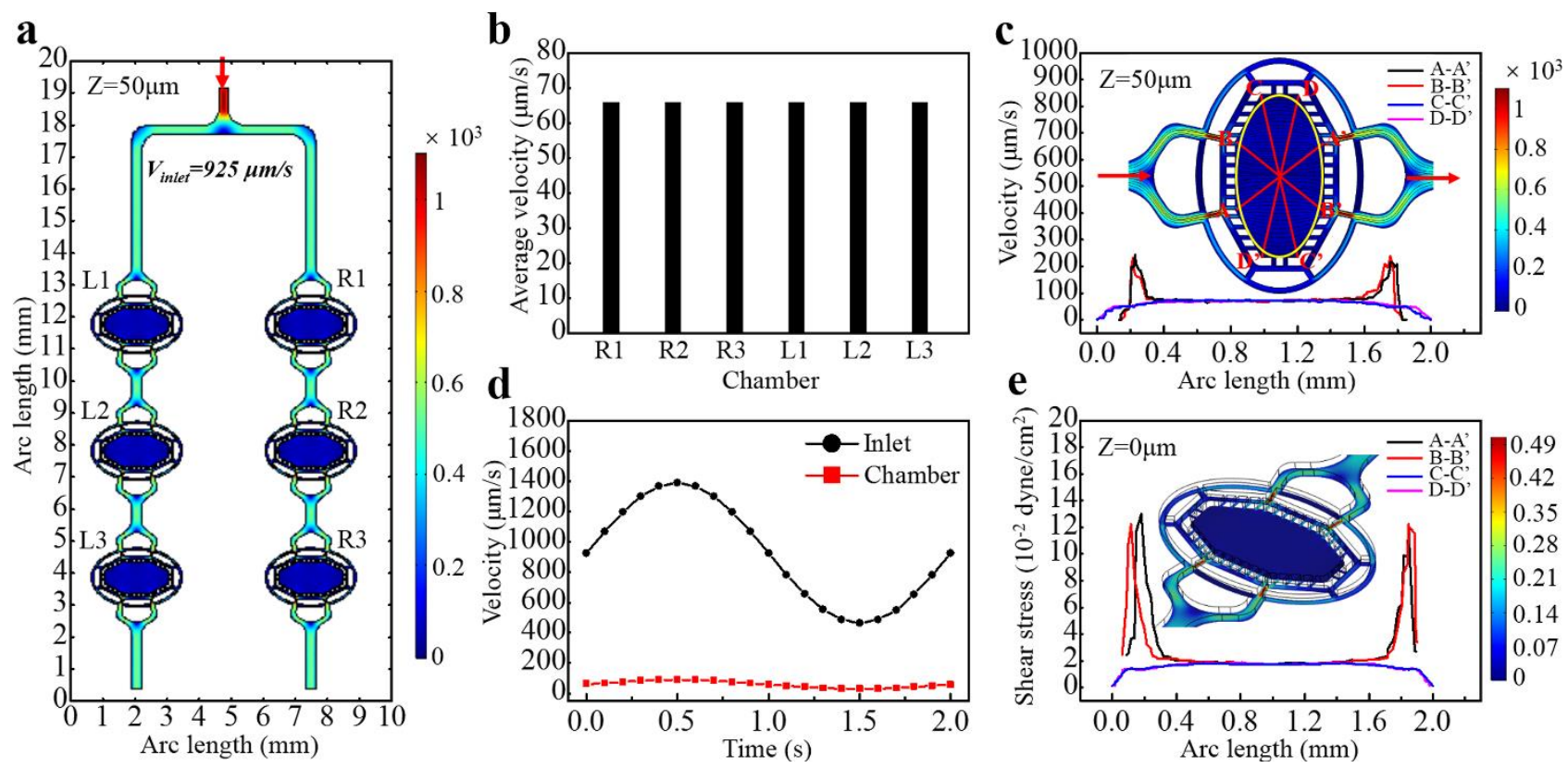

Fig. 3 Finite element simulation of fluid velocities inside the culture chambers. (a) The distribution of velocity inside the cell culturing layer at $Z=50 \mu \mathrm{m}$. (b)The area average velocities of the six chambers. (c) The simulation result of velocity field inside the R1 culture chamber, cross sections are plotted along the proximal diagonals (lines A-A' and B-B') and the distal diagonals (lines C-C' and D-D'). The yellow line is tangent to the cell culture chamber. (d) The average velocity inside R1 chamber under the sinusoidal variation velocity in medium inlet. (e) Simulation result of wall shear stress inside R1 chamber, the cross sections are same with Fig. 2c. 

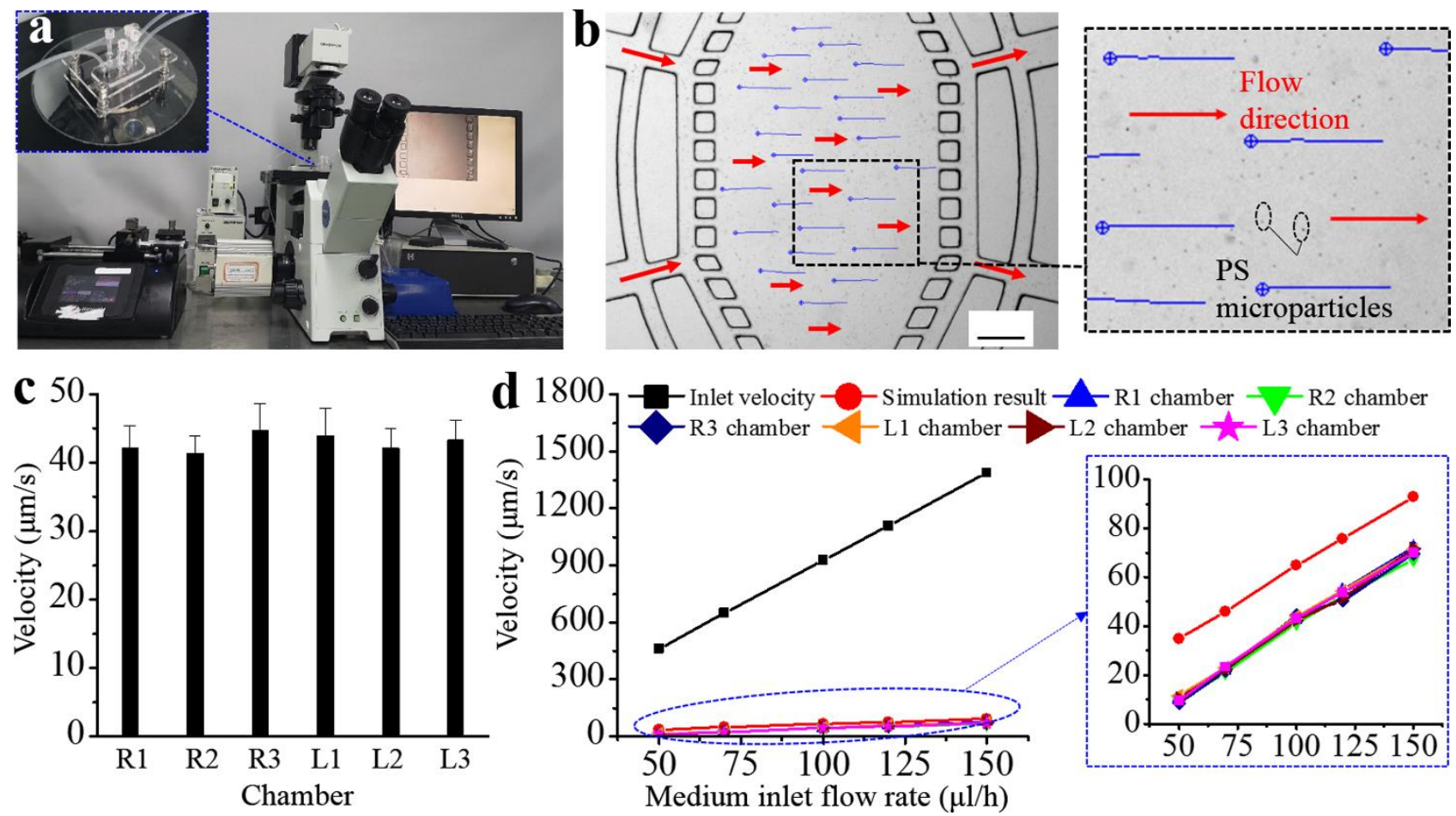

Fig. 4 The particle tracking experimental results. (a) The particle tracking experimental platform. (b) The trajectories of PS microbeads in the R3 chamber. The black dots are the PS microbeads. The red arrows represent the flow directions and the blue lines represent the trajectories of the picked PS microbeads. The scar bar is $200 \mu \mathrm{m}$. (c) The average velocities of PS microbeads in the six chambers under medium inlet flow rate of $100 \mu \mathrm{l} / \mathrm{h}$. (d) The average velocities of PS microbeads in the six chambers vary with the velocity in the medium inlet. 

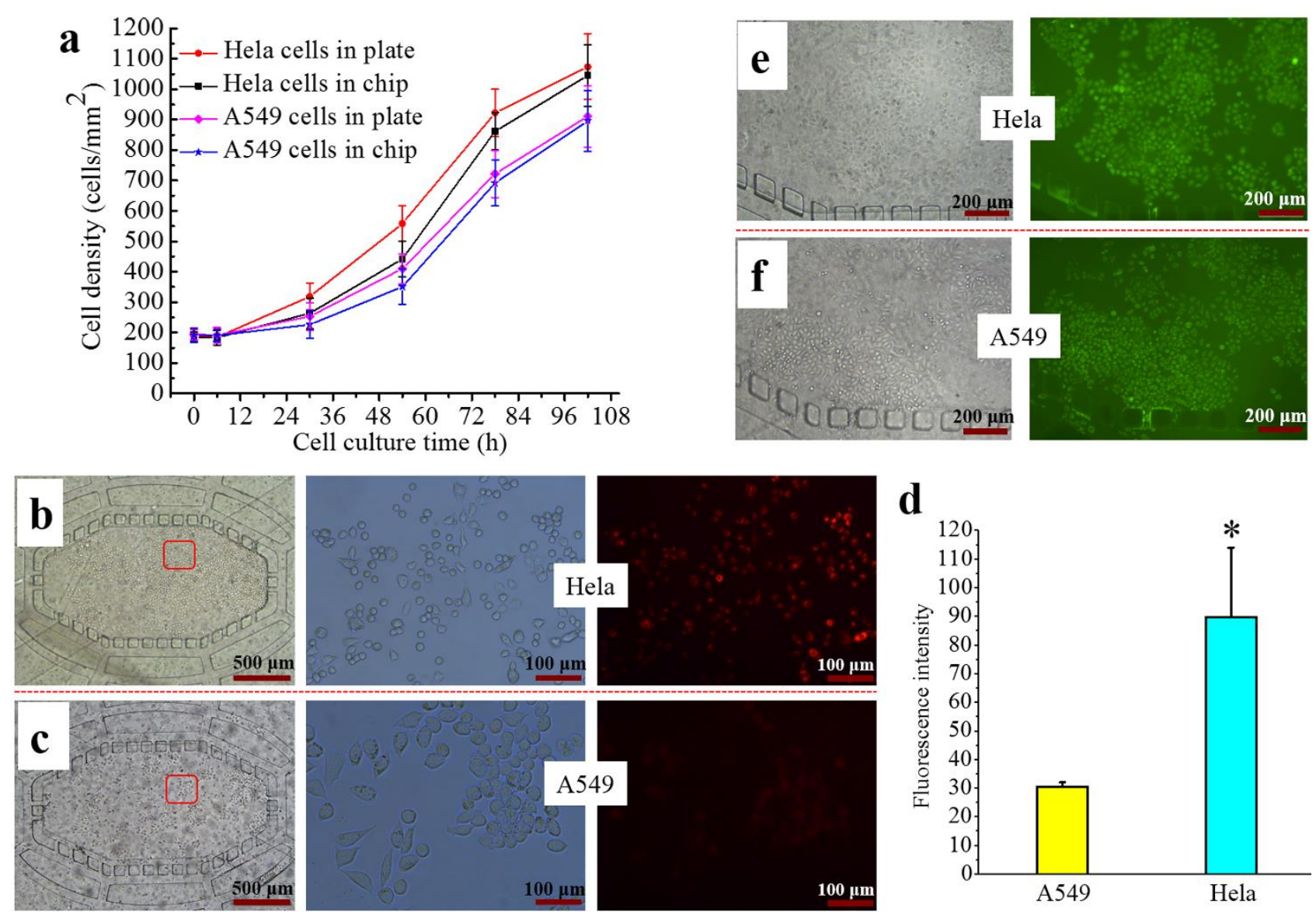

Fig. 5 Cells grow inside the microfluidic chip. (a) The densities of Hela and A549 cells inside the culture plates and microfluidic chips. (b) Distribution of folate receptors on membrance of Hela cells cultured inside the microfluidic chip. (c) Distribution of folate receptors on membrance of A549 cells cultured inside the microfluidic chip. (d) Fluorescence intensity of TRITC in Hela and A549 cells cultured in microfluidic chip. (e) Hela cells treated by FITC-FACC nanoparticles. (f) A549 cells treated by FITC-FACC nanoparticles. 

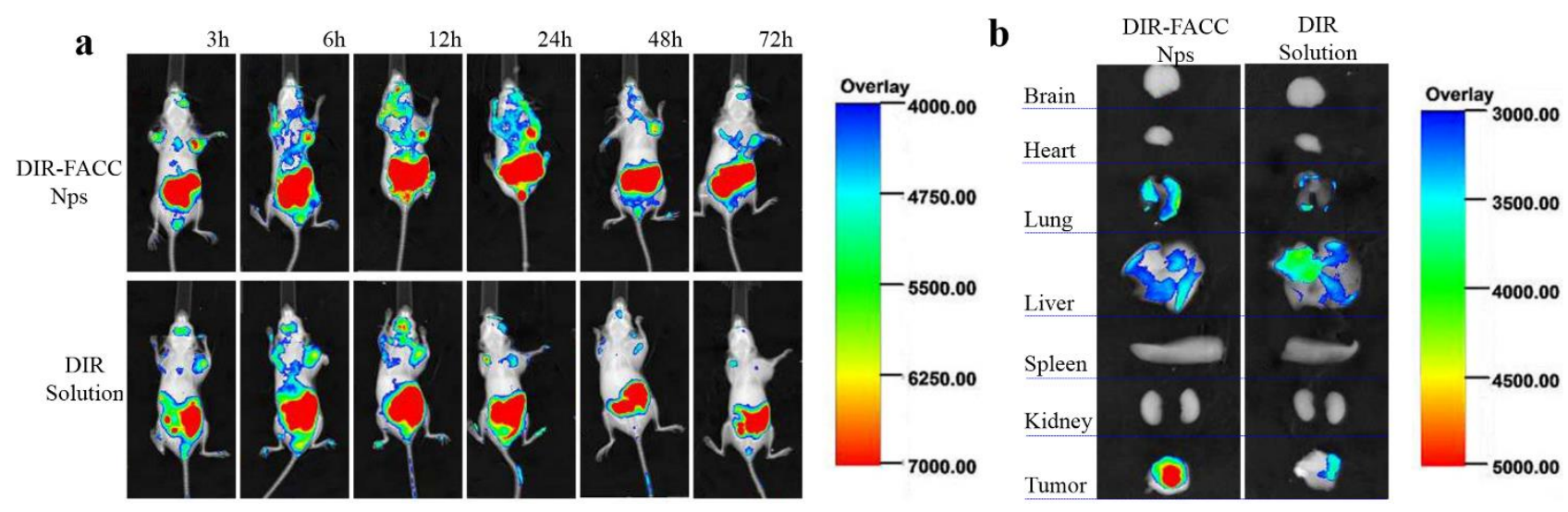

Fig. 6 In vivo targeting behavior of FACC nanoparticles. (a) Distribution of DIR-FACC nanoparticles in nude mouse varying with time. The group DIR solution is used as a comparison. (b) Distribution of DIR-FACC and DIR in different organs.

\section{References}

[1] M. Chandana, D. Manasi, J.R. Kanwar, S.K. Sahoo, Receptor mediated tumor targeting: an emerging approach for cancer therapy, Curr. Drug Delivery. 8 (2011) 45-58.

[2] D. Rosenblum, N. Joshi, W. Tao, J.M. Karp, P. Dan, Progress and challenges towards targeted delivery of cancer therapeutics, Nat. Commun. 9 (2018) 1410.

[3] R. Nasiri, J.H. Almaki, A.B. Idris, F.A.A. Majid, M. Nasiri, M. Salouti, M. Irfan, N. Amini, M. Marvibaigi, In vitro evaluation of actively targetable superparamagnetic nanoparticles to the folate receptor positive cancer cells, Mater. Sci. Eng., C. 69 (2016) 1147-1158.

[4] J. Zhang, X. Zhao, M. Xian, C. Dong, S. Shuang, Folic acid-conjugated green luminescent carbon dots as a nanoprobe for identifying folate receptor-positive cancer cells, Talanta. 183 (2018) 39-47.

[5] J. Ahn, J. Ko, S. Lee, J. Yu, Y.T. Kim, N.L. Jeon, Microfluidics in nanoparticle drug delivery: From synthesis to pre-clinical screening, Adv. Drug Delivery Rev. 128 (2018) 29-53.

[6] E.W.K. Young, D.J.Beebe, Fundamentals of microfluidic cell culture in controlled microenvironments, Chem Soc 
Rev. 39 (2010) 1036-1048.

[7] R.K. Jain, J.D. Martin, S. Triantafyllos, The role of mechanical forces in tumor growth and therapy, Annu Rev Biomed Eng. 16 (2014) 321-346.

[8] P. Joong Yull, J.B. White, W. Natalie, K. Chuan-Hsien, C. Wansik, M.E. Meyerhoff, T. Shuichi, Responses of endothelial cells to extremely slow flows, Biomicrofluidics. 5 (2011) 022211.

[9] C. Luo, X. Zhu, T. Yu, X. Luo, O. Qi, H. Ji, Y. Chen, A fast cell loading and high-throughput microfluidic system for long-term cell culture in zero-flow environments, Biotechnol. Bioeng. 101 (2008) 190-195.

[10] W.J. Polacheck, J.L. Charest, R.D. Kamm, Interstitial flow influences direction of tumor cell migration through competing mechanisms, Proc Natl Acad Sci. 108 (2011) 11115-11120.

[11] M. Swartz, M. Fleury, Interstitial flow and its effects in soft tissues, Annu Rev Biomed Eng. 9 (2007) 229-256.

[12] T. Denayer, T. Stöhr, M.V. Roy, Animal models in translational medicine: Validation and prediction, New Horizons in Translational Medicine. 2 (2014) 5-11.

[13] P.M. Valencia, O.C. Farokhzad, K. Rohit, L. Robert, Microfluidic technologies for accelerating the clinical translation of nanoparticles, Nat Nanotechnol.7 (2012) 623-629.

[14] D. Gao, H. Liu, Y. Jiang, J.M. Lin, Recent developments in microfluidic devices for in vitro cell culture for cell-biology research, Trends Anal. Chem.35 (2012) 150-164.

[15] R. Michna, M. Gadde, A. Ozkan, M. Dewitt, M. Rylander, Vascularized microfluidic platforms to mimic the tumor microenvironment, Biotechnol. Bioeng. 115 (2018) 2793-2806.

[16] J. Shao, L. Wu, J. Wu, Y. Zheng, H. Zhao, Q. Jin, J. Zhao, Integrated microfluidic chip for endothelial cells culture and analysis exposed to a pulsatile and oscillatory shear stress, Lab Chip 9 (2009) 3118.

[17] M. Mehling, S. Tay, Microfluidic cell culture, Curr. Opin. Biotechnol. 25 (2014) 95.

[18] S. Halldorsson, E. Lucumi, R. Gómez-Sjöberg, R.M.T. Fleming, Advantages and challenges of microfluidic cell culture in polydimethylsiloxane devices, Biosensors and Bioelectronics 63 (2015) 218-231. 
[19] Y. Gao, P. Li, D. Pappas, A microfluidic localized, multiple cell culture array using vacuum actuated cell seeding: integrated anticancer drug testing, Biomed. Microdevices 15 (2013) 907-915.

[20] Z. Wang, Z. Liu, L. Li, Q. Liang, Investigation into the hypoxia-dependent cytotoxicity of anticancer drugs under oxygen gradient in a microfluidic device, MICROFLUID NANOFLUID 6 (2015) 1271-1279.

[21] D. An, K. Kim, J. Kim, Microfluidic System Based High Throughput Drug Screening System for Curcumin/TRAIL Combinational Chemotherapy in Human Prostate Cancer PC3 Cells, BIOMOL THER 4 (2014) 355-362.

[22] E. Jastrzebska Jedrych, I. Grabowska-Jadach, M. Chudy, A. Dybko, Z. Brzozka, Multi-function microsystem for cells migration analysis and evaluation of photodynamic therapy procedure in coculture, BIOMICROFLUIDICS 4 (2012) 44116.

[23] E. Jastrzebska, M. Bulka, N. Rybicka, K. Zukowski, Analysis of the efficiency of photodynamic therapy using a microsystem for mono-, co- and mixed cultures, Sens. Actuator B-Chem. (2015) 1356-1365.

[24] J. Zhang, J. Wu, H. Li, Q. Chen, J. Lin, An in vitro liver model on microfluidic device for analysis of capecitabine metabolite using mass spectrometer as detector, Biosensors and Bioelectronics (2015) 322-328.

[25] M. Jarvis, M. Arnold, J. Ott, K. Pant, B. Prabhakarpandian, S. Mitragotri, Microfluidic co-culture devices to assess penetration of nanoparticles into cancer cell mass, Bioeng Transl Med. 2 (2017) 268-277.

[26] X. Ai, W. Lu, K. Zeng, C. Li, Y. Jiang, P. Tu, Microfluidic Coculture Device for Monitoring of Inflammation-Induced Myocardial Injury Dynamics, Anal. Chem. 90 (2018) 4485-4494.

[27] M. Chudy, K. Tokarska, E. Jastrzębska, M. Bułka, S. Drozdek, Ł. Lamch, K.A. Wilk, Z. Brzózka, Lab-on-a-chip systems for photodynamic therapy investigations, Biosensors and Bioelectronics (2018) 37-51.

[28] T. Zehnder, A.R. Boccaccini, R. Detsch, Biofabrication of a co-culture system in an osteoid-like hydrogel matrix, Biofabrication 9 (2017) 025016.

[29] C. Wang, J. Li, S. Sinha, A. Peterson, G.A. Grant, F. Yang, Mimicking brain tumor-vasculature microanatomical 
architecture via co-culture of brain tumor and endothelial cells in 3D hydrogels, Biomaterials 202 (2019) 35-44.

[30] A. Zuchowska, K. Marciniak, U. Bazylinska, E. Jastrzebska, K.A. Wilk, Z. Brzozka, Different action of nanoencapsulated meso-tetraphenylporphyrin in breast spheroid co-culture and mono-culture under microfluidic conditions, Sens. Actuators, B. 275 (2018) 69-77.

[31] A. Ozkan, N. Ghousifam, P.J. Hoopes, M.N. Rylander, In Vitro Vascularized Liver and Tumor Tissue Microenvironments on a Chip for Dynamic Determination of Nanoparticle Transport and Toxicity, Biotechnol. Bioeng. 116 (2019) 1201-1219.

[32] S. Nagaraju, D. Truong, G. Mouneimne, M. Nikkhah, Microfluidic Tumor-Vascular Model to Study Breast Cancer Cell Invasion and Intravasation, ADV HEALTHC MATER 9 (2018) 1701257.

[33] S. Mao, J. Zhang, H. Li, J. Lin, Strategy for signaling molecule detection by using an integrated microfluidic device coupled with mass spectrometry to study cell-to-cell communication, Anal Chem. 85 (2013) 868-876.

[34] F. Guo, J.B. French, P. Li, H. Zhao, C.Y. Chan, J.R. Fick, S.J. Benkovic, T.J. Huang, Probing cell-cell communication with microfluidic devices, Lab Chip 13 (2013) 3152-3162.

[35] J. Li, J. Wei, Y. Liu, B. Liu, T. Liu, Y. Jiang, L. Ding, C. Liu, A microfluidic design to provide a stable and uniform in vitro microenvironment for cell culture inspired by the redundancy characteristic of leaf areoles, Lab Chip. 17 (2017) 3921-3933.

[36] J. Wei, C. Liu, Y. Jiang, T. Liu, L. Chen, B. Liu, J. Li, Uniform and stable supply of medium for in-vitro cell culture using a robust chamber, J. Micromech. Microeng. 28 (2018) 065006.

[37] J. Wei, C. Liu, Y. Jiang, C. Duan, L. Chen, W. Li, B. Liu, J. Li, Microfluidic device for generating regionalized concentration gradients under a stable and uniform fluid microenvironment, J. Micromech. Microeng. 29 (2019) 15008.

[38] C. Liang, F. Meng, J. Li, C. Liu, Using CO2-laser bugle for ultrasonic bonding of thermoplastic microfluidic devices, J. Materials Processing Tech. 252 (2018) 25-33. 
[39] M.W. van der Helm, A.D. van der Meer, J.C.T. Eijkel, A. van den Berg, L.I. Segerink, Microfluidic organ-on-chip technology for blood-brain barrier research, Tissue Barriers 1 (2016) e1142493.

[40] Y. Chisti, Hydrodynamic damage to animal cells, Crit Rev Biotechnol. 21 (2001) 67-110.

[41] J. Hua, L.E. Erickson, T.Y. Yiin, L.A. Glasgow, A review of the effects of shear and interfacial phenomena on cell viability, Crit Rev Biotechnol. 13 (1993) 305-28.

[42] J.Y. Park, J.B. White, N. Walker, C. Kuo, W. Cha, M.E. Meyerhoff, S. Takayama, Responses of endothelial cells to extremely slow flows, BIOMICROFLUIDICS 2 (2011) 22211.

[43] L.C. Cheng, Y. Jiang, Y. Xie, L.L. Qiu, Q. Yang and H.Y. Lu, Novel amphiphilic folic acid-cholesterol-chitosan micelles for paclitaxel delivery, Oncotarget. 8 (2017) 3315-3326.

[44] F.Q. Hu, Cellular uptake and cytotoxicity of shell crosslinked stearic acid-grafted chitosan oligosaccharide micelles encapsulating doxorubicin, Eur. J. Pharm. Biopharm.69 (2008) 117-125.

[45] H. Oda, T. Konno, K. Ishihara, The use of the mechanical microenvironment of phospholipid polymer hydrogels to control cell behavior, Biomaterials 34 (2013) 5891-5897.

[46] H. Li, J. Ma, Z. Hou, H. Xin, Constructive role of internal noise for the detection of weak signal in cell system, Acta Phys.-Chim. Sin. 24 (2008) 2203-2208.

[47] S. Giulitti, E. Magrofuoco, L. Prevedello, N. Elvassore, Optimal periodic perfusion strategy for robust long-term microfluidic cell culture, Lab Chip 13 (2013) 4430-4441. 